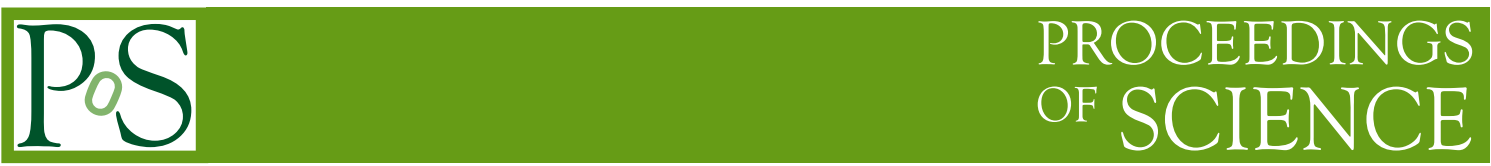

\title{
Compton Scattering and Hadron Polarizabilities
}

\author{
P.P. Martel ${ }^{* \dagger}$ \\ Johannes Gutenberg-Universität Mainz. \\ and Mount Allison University \\ E-mail: martel@uni-mainz.de
}

\begin{abstract}
The proton, at one time, was thought to be a fundamental particle. It's of course now recognized that it and its fellow hadrons are composed of quarks. This composition gives rise to parameters, called polarizabilities, that describe how this structure responds to external electric and magnetic fields. To study these polarizabilities, a Compton scattering program has been ongoing in the A2 experimental hall at the Mainz Microtron. There, a tagged Bremsstrahlung beam of either linearly or circularly polarized photons is scattered off of either unpolarized protons in a liquid hydrogen target, or polarized protons with spins transverse or longitudinal to the beam direction. Different combinations allow for the measurement of different polarization observables, using the nearly $4 \pi$ steradian coverage provided by the combination of the Crystal Ball and TAPS detectors. Combined fits of these parameters then permit an extraction of these polarizabilities, some of which had not previously been experimentally determined. These proceedings will discuss the status and outlook of the A2 Compton program.
\end{abstract}

The 9th International workshop on Chiral Dynamics

17-21 September 2018

Durham, NC, USA

\footnotetext{
* Speaker.

${ }^{\dagger}$ On behalf of the A2 Collaboration at MAMI.
} 


\section{Purpose}

Ernest Rutherford discovered the proton in 1917, just over a hundred years ago. Considering that the proton composes the majority of the visible mass in the universe, it's reasonable to expect that it's well understood at this stage. To a large degree this is true, but various puzzles remain, such as how large is its radius [1] and what makes up its spin [2]. There are still some questions regarding its interaction with an electric or magnetic field, a seemingly fundamental thing. When described by an effective Hamiltonian, at zeroth order this interaction is determined by its mass, $m$, and electric charge, $e$ :

$$
H_{\mathrm{eff}}^{(0)}=\frac{\vec{\pi}^{2}}{2 m}+e \phi,
$$

where the covariant momentum $\vec{\pi}=\vec{p}-e \vec{A}$, given momentum $\vec{p}$ and vector potential $\vec{A}$, and $\phi$ is the scalar potential [3]. At first order, the anomalous magnetic moment, $\kappa$, also plays a role:

$$
H_{\mathrm{eff}}^{(1)}=-\frac{e(1+\kappa)}{2 m} \vec{\sigma} \cdot \vec{H}-\frac{e(1+2 \kappa)}{8 m^{2}} \vec{\sigma} \cdot[\vec{E} \times \vec{\pi}-\vec{\pi} \times \vec{E}],
$$

where $\vec{H}$ and $\vec{E}$ are the magnetic and electric fields, respectively. These terms are the so-called Born terms, describing the proton as a point-like charged particle with an anomalous magnetic moment. The internal structure of the proton becomes apparent at second order:

$$
H_{\mathrm{eff}}^{(2)}=-4 \pi\left[\frac{1}{2} \alpha_{E 1} \vec{E}^{2}+\frac{1}{2} \beta_{M 1} \vec{H}^{2}\right],
$$

where $\alpha_{E 1}$ and $\beta_{M 1}$ are the electric and magnetic polarizabilities, respectively [4]. The effect that these scalar polarizabilities describe is visualized in Fig. 1. The scalar polarizabilities for
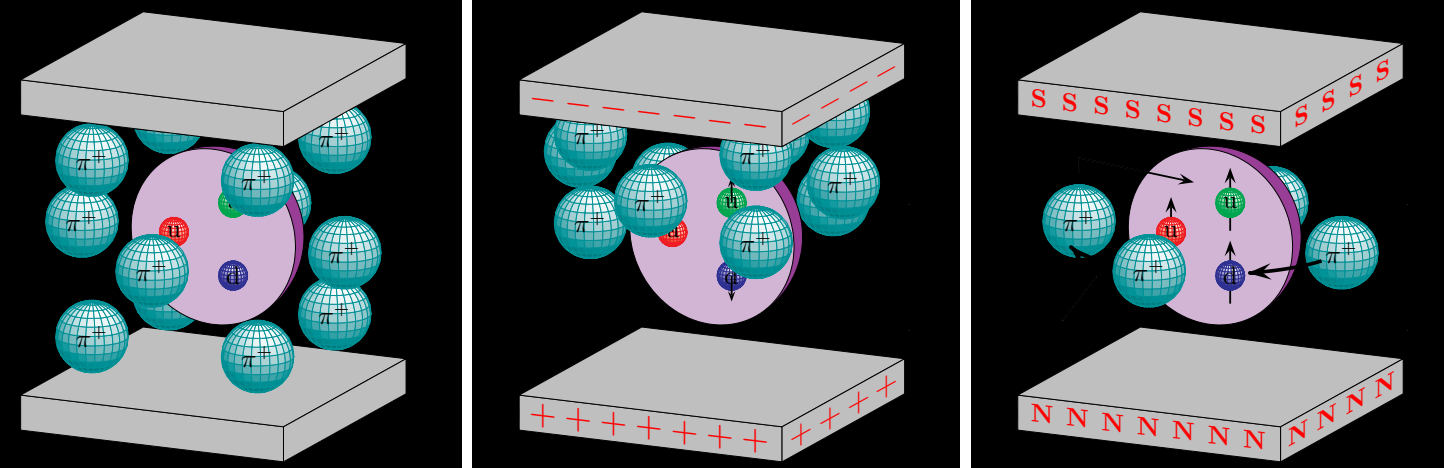

Figure 1: The left figure depicts a proton, with its constituent quarks and sea of virtual pions, placed between two parallel plates. The middle figure shows the application of an electric field across these plates, which results in a 'stretching' of the proton. The right figure shows the application of a magnetic field, which results in an 'alignment' of the proton.

the proton have been experimentally determined, though the Particle Data Group [5] numbers are averages of various extractions, some of which used the same data sets, among which there are inconsistencies [6]. 
Going higher in energy, the response of the proton spin, denoted by the Pauli matrices $\vec{\sigma}$, to an electric or magnetic field becomes important. This is described by four terms:

$$
H_{\mathrm{eff}}^{(3)}=-4 \pi\left[\frac{1}{2} \gamma_{E 1 E 1} \vec{\sigma} \cdot(\vec{E} \times \dot{\vec{E}})+\frac{1}{2} \gamma_{M 1 M 1} \vec{\sigma} \cdot(\vec{H} \times \dot{\vec{H}})-\gamma_{M 1 E 2} E_{i j} \sigma_{i} H_{j}+\gamma_{E 1 M 2} H_{i j} \sigma_{i} E_{j}\right],
$$

where the $\gamma_{S}$ are parameters called the spin polarizabilities, with $\gamma_{E 1 M 2}$, for instance, representing the excitation by a magnetic quadrupole and a de-excitation by an electric dipole. As of 2012, the four spin polarizabilities had not been individually extracted via experiments. However, two linear combinations of them had experimental determinations. The first is the forward spin polarizability:

$$
\gamma_{0}=-\gamma_{E 1 E 1}-\gamma_{E 1 M 2}-\gamma_{M 1 E 2}-\gamma_{M 1 M 1}=(-1.0 \pm 0.08) \times 10^{-4} \mathrm{fm}^{4},
$$

determined at MAMI and ELSA through the GDH experiments [7, 8], and the second is the backward spin polarizability:

$$
\gamma_{\pi}=-\gamma_{E 1 E 1}-\gamma_{E 1 M 2}+\gamma_{M 1 E 2}+\gamma_{M 1 M 1}=(8.0 \pm 1.8) \times 10^{-4} \mathrm{fm}^{4},
$$

determined with dispersive fits to back-angle Compton scattering [9]. It should be noted that this value for $\gamma_{\pi}$ is only the dispersive part, and does not include the $\pi^{0}$-pole term, $\gamma_{\pi}^{\pi^{0} \text {-pole }}=-46.7 \times$ $10^{-4} \mathrm{fm}^{4}[10]$.

Adding or subtracting those two relations from one another can produce the following:

$$
\gamma_{E 1 M 2}=-\gamma_{E 1 E 1}-\frac{1}{2} \gamma_{0}-\frac{1}{2} \gamma_{\pi},
$$

and

$$
\gamma_{M 1 E 2}=-\gamma_{M 1 M 1}-\frac{1}{2} \gamma_{0}+\frac{1}{2} \gamma_{\pi}
$$

Given these two combinations, a basis can be constructed of $\gamma_{E 1 E 1}, \gamma_{M 1 M 1}, \gamma_{0}$, and $\gamma_{\pi}$, reducing the problem to two unknown, and two uncertain, terms.

To improve the situation, a program of Compton scattering experiments was envisioned to measure several polarization observables. The first two are double-polarized asymmetries with a circularly polarized photon beam incident on a polarized target:

$$
\Sigma_{2 T}=\frac{1}{P_{\gamma} P_{T}} \times \frac{N_{+T}^{R}-N_{+T}^{L}}{N_{+T}^{R}+N_{+T}^{L}}=\frac{1}{P_{\gamma} P_{T}} \times \frac{N_{-T}^{L}-N_{-T}^{R}}{N_{-T}^{L}+N_{-T}^{R}}=\frac{1}{P_{\gamma} P_{T}} \times \frac{N_{-T}^{L}-N_{-T}^{R}}{N_{-T}^{L}+N_{-T}^{R}},
$$

where $N_{+/-T}^{R / L}$ is the number of events with a right/left helicity beam and a positively/negatively polarized target, and where $P_{\gamma}$ and $P_{T}$ are the magnitudes of the beam and target polarization, respectively, with $T$ being $x$ or $y$ for a transversely or longitudinally polarized target, respectively. The third is a linearly polarized photon beam incident on an unpolarized target:

$$
\Sigma_{3}=\frac{1}{P_{\gamma}} \times \frac{N_{\|}-N_{\perp}}{N_{\|}+N_{\perp}}
$$

where $N_{\| / \perp}$ is the number of events with a beam parallel/perpendicular to the scattering plane. These asymmetries are then fitted with values provided by Dispersion Theory (HDPV) [11] and Baryon Chiral Perturbation Theory (B $\chi$ PT) [12] calculations to extract the spin polarizabilities. 


\section{Method}

Performing these Compton scattering experiments using polarized photon beams and both polarized and unpolarized targets, within an energy region that exhibited sensitivity to the polarizabilities, limits the possibilities. One such possibility, which is discussed here, is the A2 real photon facility at the Mainz Microtron (MAMI).

MAMI is a cascade of racetrack microtrons that can provide electron beams from $180 \mathrm{MeV}$ to $1.6 \mathrm{GeV}$, in steps of approximately $15 \mathrm{MeV}[13,14]$. When required, the beam can additionally be longitudinally polarized by irradiating a GaAsP (II-V semiconductor) cathode with circularly polarized laser light [15]. This electron beam is then directed into the A2 hall, where it strikes a radiator to produce Bremsstrahlung photons with a distribution of roughly $1 / E_{\gamma}$ with energies up to the initial electron energy. If the electron is longitudinally polarized there will be a helicity transfer to the outgoing photon, resulting in a circular polarization. If the radiator is a diamond, the crystalline structure can be oriented to produce coherent Bremsstrahlung, enhancing certain regions of the distribution based on the lattice vectors. This enhancement also results in a linear polarization of the photon beam, with the plane of polarization dependent upon the orientation of the diamond lattice with respect to the lab frame [16].
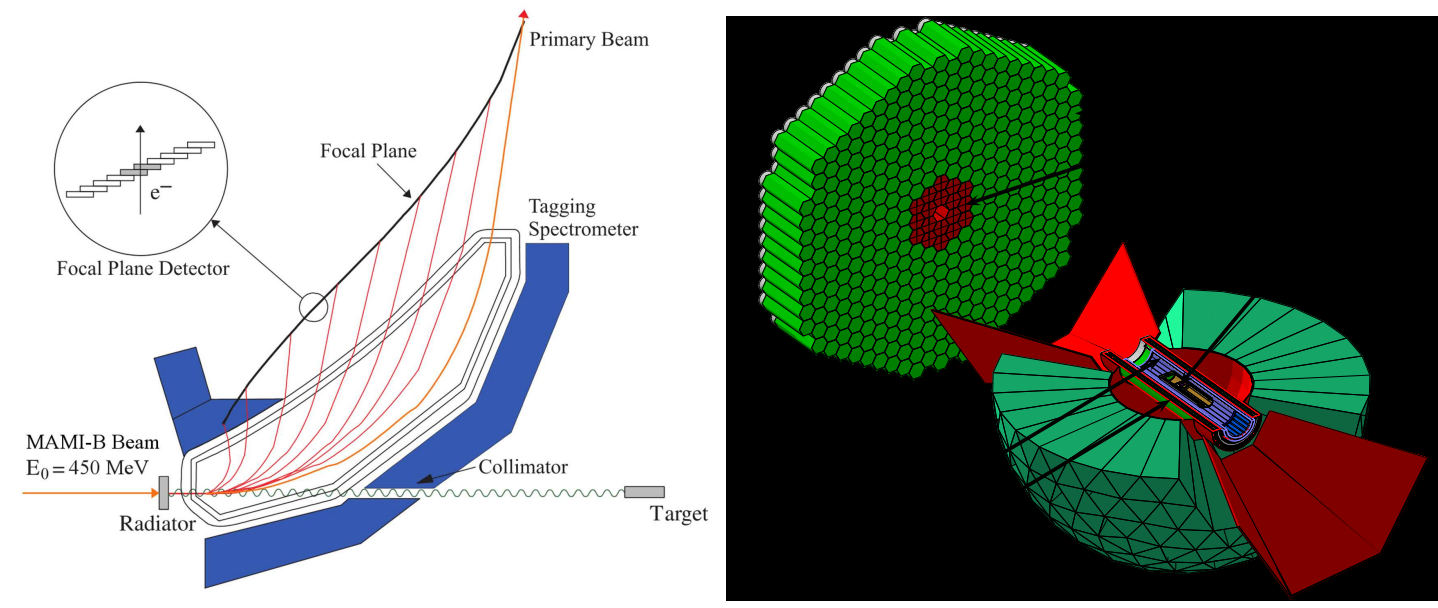

Figure 2: The detectors used in the A2 hall at MAMI. The left shows a diagram of the tagging system, and the right shows a diagram of the Crystal Ball and TAPS systems.

After the radiator, the new photon beam passes through a set of lead collimators before traveling on to the target, whereas the electron beam is bent in a tagging spectrometer magnet, as shown in Fig. 2. The magnetic field is set such that the primary electron beam is directed into a beam dump while a residual electron, having lost energy in the production of its photon, will be bent more severely, and for the majority into a ladder of detectors along the focal plane of the spectrometer. Determining the electron path in this way permits the tagging of the photon energy [17]. Until September 2017 the tagger covered 5-95\% of the residual electron energy range in 352 steps. At this point the tagger underwent an upgrade which, while maintaining the coverage, increased the number of channels to 408. Along with improved electronics this enabled a several-fold increase to the permissible flux. 
The A2 setup has various targets available such as liquid hydrogen, deuterium, and helium; a range of solid targets; and a polarized proton or deuteron target. The polarized target is a Frozen Spin Target (FST), which uses Dynamic Nuclear Polarization (DNP) to perform spin flips between the electrons and protons in the material through irradiation of microwaves with a frequency of the difference, or sum, of their Larmor frequencies [18]. The frozen spin component involves cooling the polarized target to approximately $25 \mathrm{mK}$ and energizing a holding coil located just outside the dilution region. This combination permits for high degrees of polarization (up to 90\% for protons) with relaxation times of over 1000 hours [19].

Detection of the final state particles is performed with the Crystal Ball (CB) and Two-Arms Photon Spectrometer (TAPS) systems. The CB is composed of $672 \mathrm{NaI}$ crystals arranged in a nearly spherical icosahedron, and covers a polar angular range of $20-160^{\circ}$ [20]. TAPS is composed of $366 \mathrm{BaF} 2$ and $72 \mathrm{PbWO} 4$ crystals arranged in a hexagonal wall, and covers the downstream hole of the CB [21]. All told, CB/TAPS covers approximately $96 \%$ of $4 \pi$. Each system includes a set of detectors for performing particle identification. In the case of the CB, this is done via a barrel of 24 scintillator paddles (PID) and a pair of Multi-Wire Proportional Chambers (MWPCs), all nestled within each other and arranged along the beam axis around the target nose [22]. In the case of TAPS, this is done via a wall of hexagonal scintillator plates (Veto), one for each BaF2 element and one for each set of four $\mathrm{PbWO} 4$ elements.

\section{Results}

The data used to produce the results presented here were taken over the course of several years, due to the varying target requirements. Data for the first observable, $\Sigma_{2 x}$, were taken with a transversely polarized target in September 2010 and then further in February 2011 [23]. The analysis of these data produced asymmetries for $E_{\gamma}=273-303 \mathrm{MeV}$, which are shown in Fig. 3. The plots make use of Eq. 1.7 and Eq. 1.8 to reduce the problem to two purely unknowns, $\gamma_{E 1 E 1}$ and $\gamma_{M 1 M 1}$. After fixing one of these unknowns to a given number, here chosen as the HDPV dispersion prediction, the other unknown is varied about its predicted value. The observable of interest is then calculated in the HDPV framework using those values, with each setting represented by one of the colored bands in these plots. The width of each band is a result of additionally allowing the values for $\alpha_{E 1}, \beta_{M 1}, \gamma_{0}$, and $\gamma_{\pi}$ to vary by their associated errors. The sensitivity of a specific observable to a given spin polarizability can be visually inferred from these plots. A large separation of the centroid of the bands indicates a high sensitivity to the varying spin polarizabilities. Overlapping bands indicate a higher sensitivity to the mixed terms, $\gamma_{E 1 M 2}$ or $\gamma_{M 1 E 2}$.

However, a visual representation of this form is only useful as a guide. A proper extraction of the polarizabilities requires fitting the data within the theoretical framework while allowing all of the polarizabilities to vary. Constraints, such as $\gamma_{0}$, can be added as desired. Fitting just the $\Sigma_{2 x}$ data, making use of all of the constraints, is possible, but naturally results in large uncertainties for everything except $\gamma_{E 1 E 1}$. While the other two observables, noted earlier, were being measured and analyzed, data already existed on the beam asymmetry $\Sigma_{3}$ from the LEGS collaboration [24]. These two data sets, with the use of the $\gamma_{0}$ and $\gamma_{\pi}$ constraints, provided an extraction of the two independent spin polarizabilities of $\gamma_{E 1 E 1}=-3.5 \pm 1.2 \times 10^{-4} \mathrm{fm}^{4}$ and $\gamma_{M 1 M 1}=3.2 \pm 0.9 \times 10^{-4} \mathrm{fm}^{4}$ [25]. 

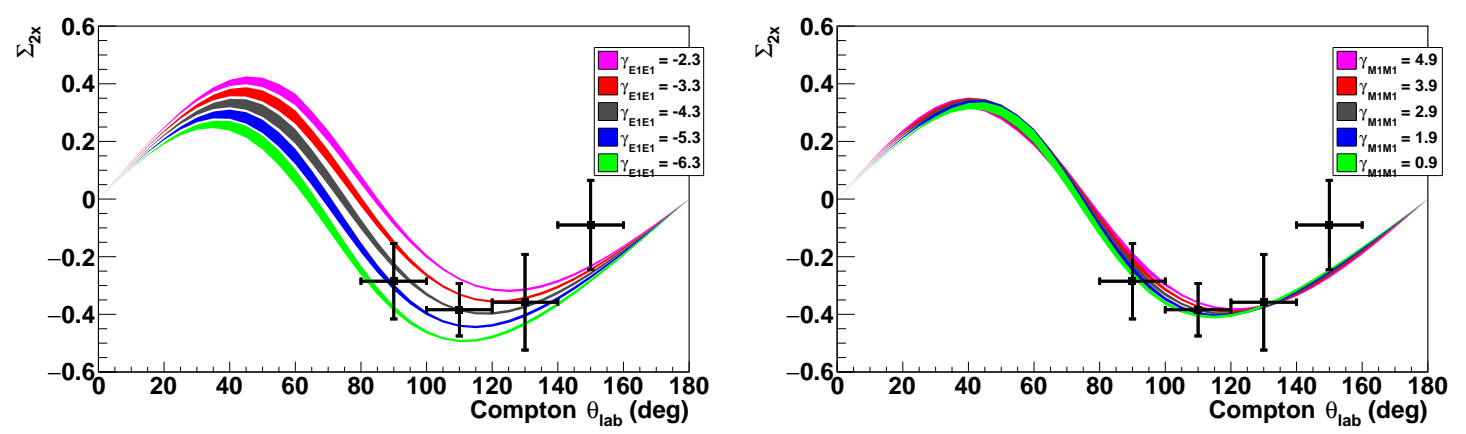

Figure 3: Beam-target asymmetry with a transversely polarized target and circularly polarized beam for $E_{\gamma}=273-303 \mathrm{MeV}$. On the left, the bands show the result of fixing $\gamma_{M 1 M 1}=$ to $2.9 \times 10^{-4} \mathrm{fm}^{4}$ and varying $\gamma_{E 1 E 1}$ between -6.3 and $-2.3 \times 10^{-4} \mathrm{fm}^{4}$. On the right, the bands show the result of fixing $\gamma_{E 1 E 1}=$ to $-4.3 \times 10^{-4} \mathrm{fm}^{4}$ and varying $\gamma_{M 1 M 1}$ between 0.9 and $4.9 \times 10^{-4} \mathrm{fm}^{4}$. The width of each band is a result of additionally allowing the values for $\alpha_{E 1}, \beta_{M 1}, \gamma_{0}$, and $\gamma_{\pi}$ to vary by their associated errors.

In May 2014 and June 2015, the A2 collaboration took data on $\Sigma_{2 z}$. The analysis, which was undergoing corrections as of the time of this workshop, has since been finalized, and a paper is in the last stages of an internal review. The extraction with these data is expected to reduce the errors on the spin polarizabilities by a factor of two. These data are shown in Fig. 4, with the same theoretical treatment as in Fig. 3.

The initial goal of the Compton program at MAMI was to produce a set of observables that would enable an independent extraction of the spin polarizabilities. For that reason, despite the existence of the $\Sigma_{3}^{\text {LEGS }}$ data, the A2 collaboration also collected data for the beam asymmetry in December 2012 [16]. The analysis for this is essentially finished, with only a study of the systematics in the determination of the degree of linear polarization of the photon beam still to be finalized. These data are shown in Fig. 5, with the same theoretical treatment as in Fig. 3.

While the data do not provide a statistical improvement over the LEGS data, they do provide a consistency check, and allow for a totally independent extraction of the spin polarizabilities. Fig. 6 shows the result of fitting to either the $\Sigma_{3}^{\mathrm{LEGS}}$ data or the $\Sigma_{3}^{\mathrm{MAMI}}$ data.

Data on the liquid hydrogen target with the same MAMI energy were taken in May and September 2018 to study the $\pi^{0}$ transition form factor. The diamond radiator was utilized with the same setup as in the December 2012 run; while linear polarization was not needed for the $\pi^{0}$ TFF, the enhancement from it provided an increase to the photon flux in the region of interest. These data are being analyzed with the expectation of notably improving the $\Sigma_{3}^{\mathrm{MAMI}}$ data, and with it the SP extraction.

Taking a step back from the spin polarizabilities, it was evident that further work could be done investigating the scalar polarizabilities, for which an additional program of Compton scattering measurements was undertaken at MAMI. The goal was to measure both the unpolarized cross section and again the beam asymmetry, though at energies below the pion production threshold, and fit the theoretical frameworks to them, with additional support from Heavy Baryon Chiral Perturbation Theory colleagues [6]. A test run was performed in June 2013, the results of which were published in 2017 [26]. While the test run was enough to extract its own value of $\alpha_{E 1}$ and, notably, $\beta_{M 1}$, the remainder of the approved beam was taken in several runs in November 2017 , 

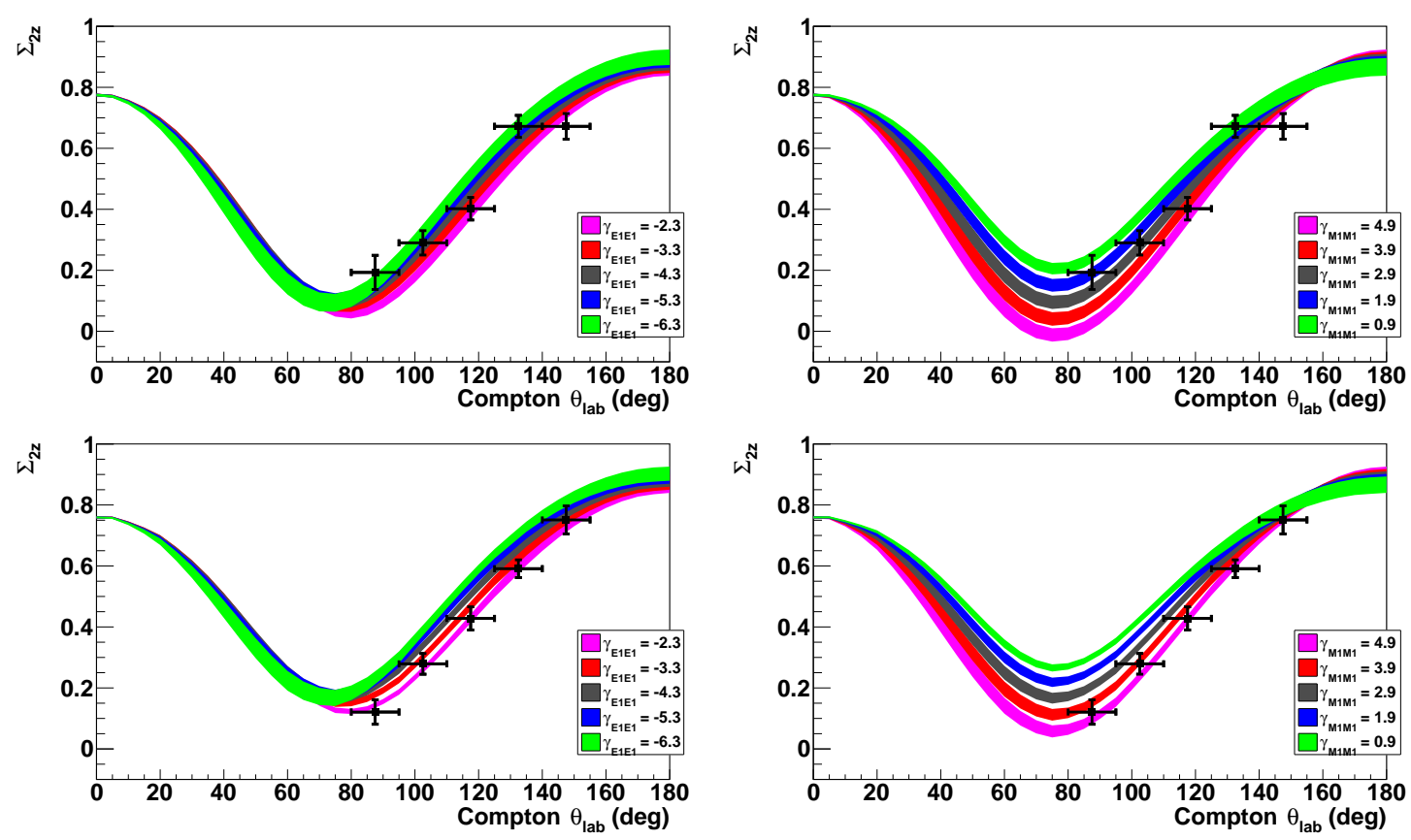

Figure 4: Beam-target asymmetry with a longitudinally polarized target and circularly polarized beam for $E_{\gamma}=265-285 \mathrm{MeV}$ (top) and $E_{\gamma}=285-305 \mathrm{MeV}$ (bottom). On the left, the bands show the result of fixing $\gamma_{M 1 M 1}=$ to $2.9 \times 10^{-4} \mathrm{fm}^{4}$ and varying $\gamma_{E 1 E 1}$ between -6.3 and $-2.3 \times 10^{-4} \mathrm{fm}^{4}$. On the right, the bands show the result of fixing $\gamma_{E 1 E 1}=$ to $-4.3 \times 10^{-4} \mathrm{fm}^{4}$ and varying $\gamma_{M 1 M 1}$ between 0.9 and $4.9 \times 10^{-4} \mathrm{fm}^{4}$. The width of each band is a result of additionally allowing the values for $\alpha_{E 1}, \beta_{M 1}, \gamma_{0}$, and $\gamma_{\pi}$ to vary by their associated errors.

March 2018, and July 2018. These data combined will represent a marked improvement in the statistical and systematic errors over the test run, and will permit an independent extraction of $\alpha_{E 1}$ and $\beta_{M 1}$ at the uncertainty level of the current values by the PDG [5].

\section{Future}

The extraction of the spin polarizabilities, through analysis of the three asymmetries in the $\Delta(1232)$ region, is at this point limited by the energy range kinematically accessible in the two polarized target observables. The reason for this is the large backgrounds from heavy nuclei in the FST. The carbon and oxygen of the butanol, along with the helium bath it is immersed in, produce both coherent and incoherent Compton scattering events, as well as coherent and incoherent $\pi^{0}$ production. While this background should be completely removable by separate running on a carbon target (with a density and thickness to account for the oxygen) in a helium bath, the rate of Compton scattering on the proton is dwarfed by these backgrounds, resulting in large uncertainties after such a subtraction. Therefore, instead of relying on just the reconstruction of the Compton scattered photon, detection of the recoil proton is required. While the proton suffers a significant amount of energy loss in traveling to the detectors, its vector is still useful for a kinematic confirmation of the Compton event. Unfortunately this loss of energy results in a lower limit to the accessible energy range, as shown in Fig. 7. 

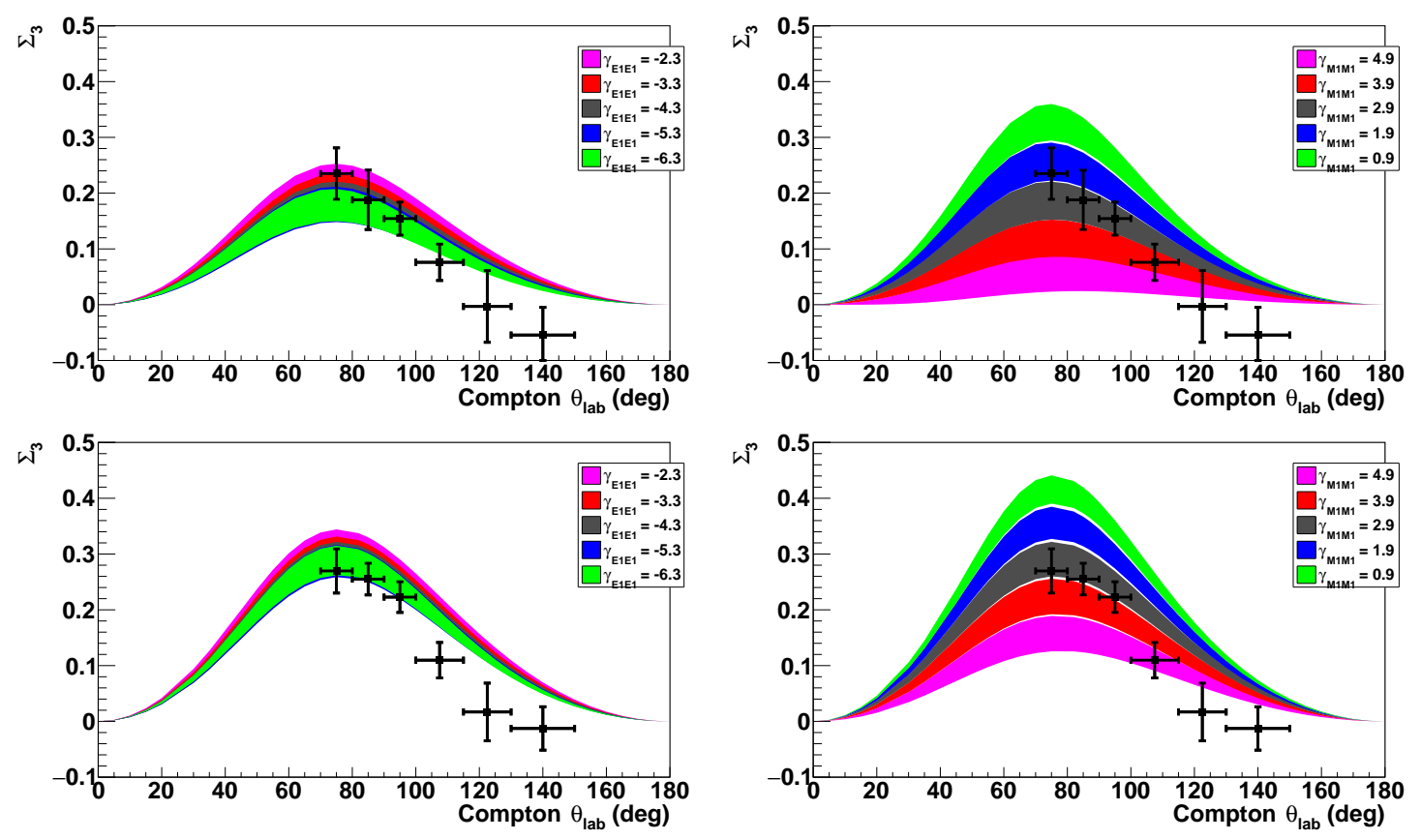

Figure 5: Beam asymmetry with a unpolarized target and linearly polarized beam for $E_{\gamma}=267-287 \mathrm{MeV}$ (top) and $E_{\gamma}=287-307 \mathrm{MeV}$ (bottom). On the left, the bands show the result of fixing $\gamma_{M 1 M 1}=$ to $2.9 \times 10^{-4} \mathrm{fm}^{4}$ and varying $\gamma_{E 1 E 1}$ between -6.3 and $-2.3 \times 10^{-4} \mathrm{fm}^{4}$. On the right, the bands show the result of fixing $\gamma_{E 1 E 1}=$ to $-4.3 \times 10^{-4} \mathrm{fm}^{4}$ and varying $\gamma_{M 1 M 1}$ between 0.9 and $4.9 \times 10^{-4} \mathrm{fm}^{4}$. The width of each band is a result of additionally allowing the values for $\alpha_{E 1}, \beta_{M 1}, \gamma_{0}$, and $\gamma_{\pi}$ to vary by their associated errors.
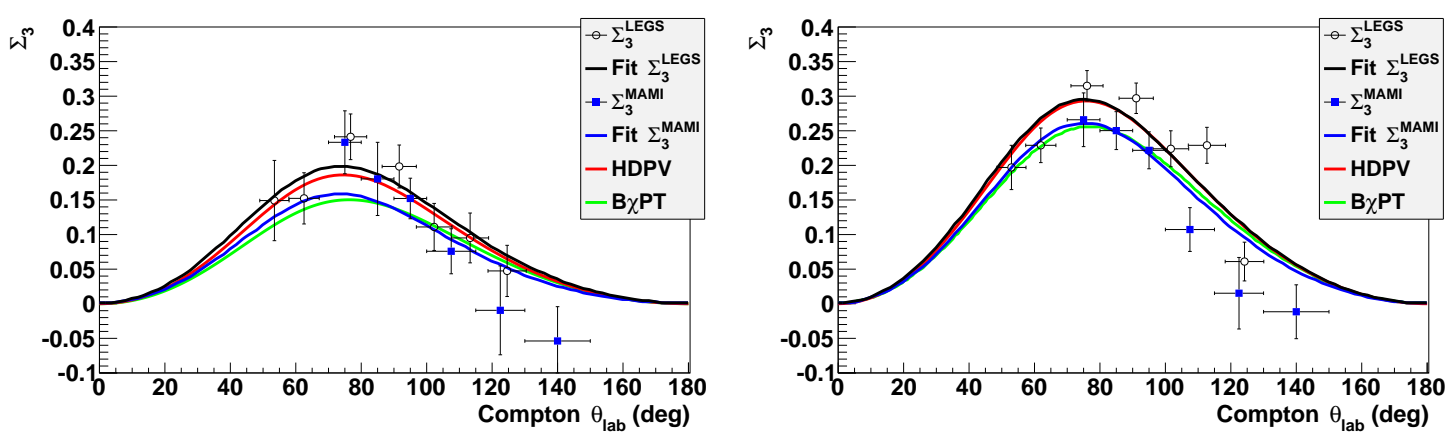

Figure 6: Beam asymmetry with a unpolarized target and linearly polarized beam for $E_{\gamma}=267-287 \mathrm{MeV}$ (left) and $E_{\gamma}=287-307 \mathrm{MeV}$ (right). The open circles are the LEGS data [24], with the black curves showing the HDPV fit to that data, and the filled squares are the MAMI data, with the blue curves showing the HDPV fit to that data. The red and green curves are the HDPV result using the HDPV and B $\chi$ PT predicted values for the spin polarizabilities. 

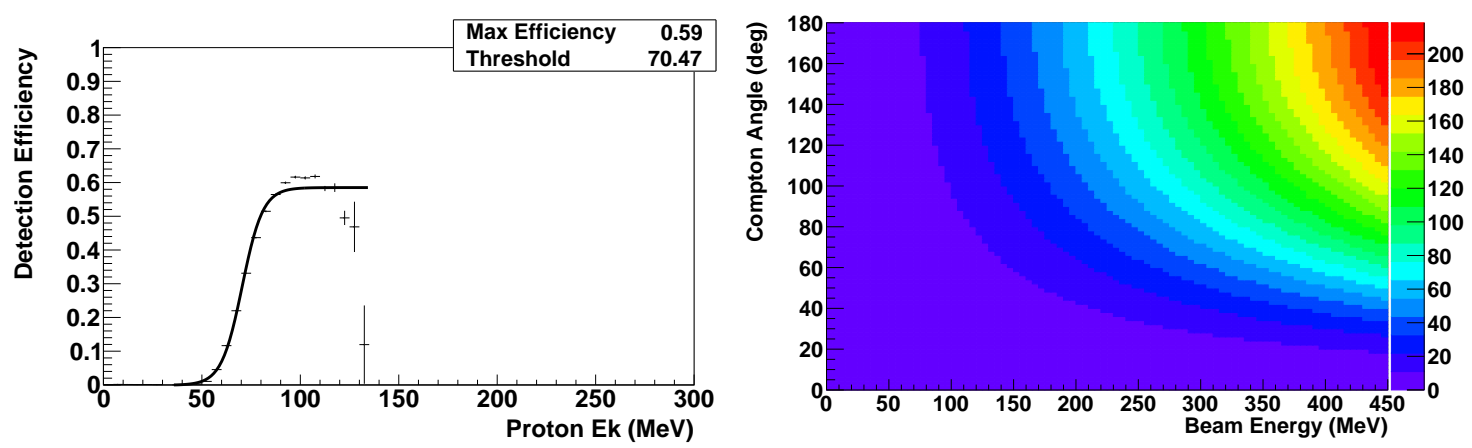

Figure 7: Restrictions to energy range due to kinematic constraints. The figure on the left shows the reconstructed proton detection efficiency in the CB as a function of its kinetic energy. The figure on the right shows the kinematics of Compton scattering, with the incoming beam energy on the $\mathrm{x}$-axis, the scattered angle on the y-axis, and the recoil proton energy on the z-axis, here depicted as a color contour. The threshold of detection of $70 \mathrm{MeV}$ from the left plot then represents a region of detection above and to the right of the lightest blue band.

To access energies below $265 \mathrm{MeV}$ with the polarized target, one needs to address this proton loss. This can be done by the use of an active target, where the passage of the recoiling proton generates scintillation light directly in the target material itself. Detecting this scintillation light would enable a rejection of all coherent events on non-hydrogen nuclei in the target, and therefore a significant reduction of the background. A prototype of an active polarized target was installed, polarized, and irradiated in June 2016 [27]. This test was successful, in-so-far as the target could
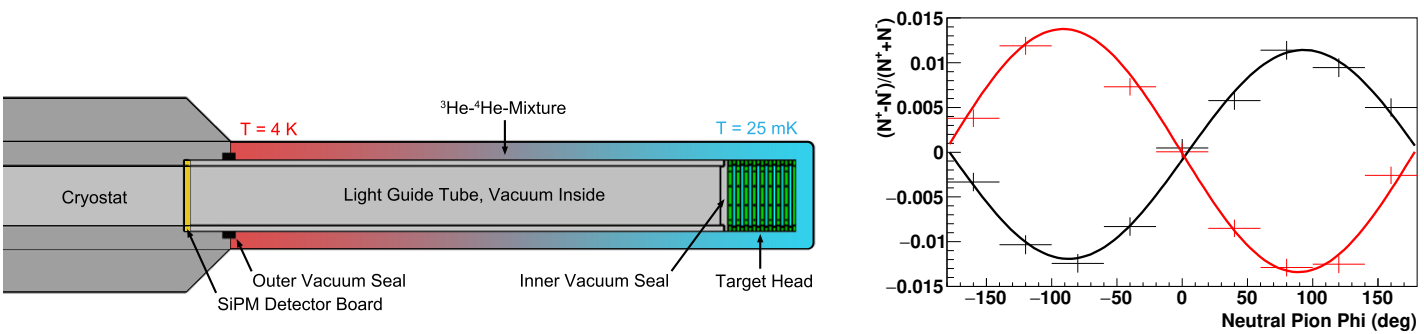

Figure 8: A schematic for an active polarized target insert is shown on the left [27]. A rough analysis of a target asymmetry from $\pi^{0}$ photoproduction is shown on the right, verifying the polarization of the target.

be polarized up to $50 \%$, which was confirmed with a rough target asymmetry analysis on $\pi^{0}$ photoproduction as shown, along with a schematic of the target, in Fig. 8. The light collection capability of the target was also demonstrated, and improvements of the design are underway to maximize this.

Beyond these improvements to the proton data, the A2 collaboration is also turning to study these polarizabilities for the neutron, with experiments on an active helium target and on an active polarized deuterium target in development.

\section{Conclusion}

The A2 collaboration at MAMI has undertaken a program of Compton scattering experiments 
using polarized photon beams on polarized and unpolarized targets in order to extract all four spin polarizabilities of the proton for the first time, as well as improve upon the extraction of the scalar polarizabilities of the proton. As this completes, the next round of experiments, now focused on the neutron, will begin.

\section{References}

[1] R. Pohl et al. (CREMA), Nature 466, 213 (2010).

[2] J. Ashman et al. (EMC), Phys. Lett. B 206, 364 (1988).

[3] M.I. Levchuk and A.I. L'vov, Nucl. Phys. A 674, 449-492 (2000).

[4] B. Pasquini, D. Drechsel, and M. Vanderhaeghen, Phys. Rev. C 76, 015203 (2007).

[5] M. Tanabashi et al. (Particle Data Group), Phys. Rev. D 98, 030001 (2018).

[6] H.W. Grießhammer, J.A. McGovern, D.R. Phillips, and G. Feldman, Prog. Part. Nucl. Phys. 67, 841-897 (2012).

[7] J. Ahrens et al. (GDH/A2), Phys. Rev. Lett. 87, 022003 (2001).

[8] H. Dutz et al. (GDH), Phys. Rev. Lett. 91, 192001 (2003).

[9] M. Camen et al. (A2), Phys. Rev. C 65, 032202 (2002).

[10] M. Schumacher, Part. Nucl. Phys. 55, 567-646 (2005).

[11] B.R. Holstein, D. Drechsel, P. Pasquini, and M. Vanderhaeghen, Phys. Rev. C 61, 034316 (2000).

[12] V. Lensky and V. Pascalutsa, Eur. Phys. J. C 65, 207 (2010).

[13] A. Jankowiak, Eur. Phys. J. A 28, s01 (2006).

[14] K.-H. Kaiser et al., Nucl. Instrum. Methods Phys. Res. A, 593, 159 (2008).

[15] K. Aulenbacher et al., Nucl. Instrum. Methods A 391, 3 (1997).

[16] C. Collicott, Ph.D. thesis, Dalhousie University (2015).

[17] J. McGeorge, J. Kellie, et al., Eur. Phys. J. A 37, 129 (2008).

[18] D. G. Crabb and W. Meyer, Annu. Rev. Nucl. Part. Sci. 47, 67 (1997).

[19] A. Thomas, Eur. Phys. J. Special Topics 198, 171 (2011).

[20] A. Starostin et al. (CB), Phys. Rev. C 64, 055205 (2001).

[21] R. Novotny, IEEE Trans. Nucl. Sci. 38, 379 (1991).

[22] C.M. Tarbert et al. (CB@MAMI/A2), Phys. Rev. Lett. 100, 132301 (2008).

[23] P.P. Martel, Ph.D. thesis UMass Amherst (2013).

[24] G. Blanpied et al. (LEGS), Phys. Rev. C 64, 025203 (2001).

[25] P.P. Martel, R. Miskimen, et al. (A2), Phys. Rev. Lett. 114, 112501 (2015).

[26] V. Sokhoyan, E.J. Downie, E. Mornacchi, J.A. McGovern, N. Krupina, et al. (A2), Eur. Phys. J. A 53, 14 (2017).

[27] M. Biroth, P. Achenbach, E. Downie, and A. Thomas (A2), in proceedings of PSTP, POS (PSTP2015) 005 (2015). 